\title{
Linx
}

Revue des linguistes de l'université Paris X Nanterre

$72 \mid 2015$

Former à l'écrit universitaire, un terrain pour la linguistique?

\section{Anaphores démonstratives dans les écrits d'étudiants de Master : comparaison avec les pratiques expertes}

Françoise Boch et Fanny Rinck

\section{OpenEdition}

\section{Journals}

Édition électronique

URL : http://journals.openedition.org/linx/1631

DOI : 10.4000/linx.1631

ISSN : 2118-9692

\section{Éditeur}

Presses universitaires de Paris Nanterre

Édition imprimée

Date de publication : 1 septembre 2015

Pagination : 131-150

ISSN : 0246-8743

Référence électronique

Françoise Boch et Fanny Rinck, « Anaphores démonstratives dans les écrits d'étudiants de Master comparaison avec les pratiques expertes », Linx [En ligne], 72 | 2015, mis en ligne le 01 mars 2016, consulté le 01 mai 2019. URL : http://journals.openedition.org/linx/1631 ; DOI : 10.4000/linx.1631 


\title{
Anaphores démonstratives dans les écrits d'étudiants de Master : comparaison avec les pratiques expertes.
}

\author{
Françoise BOCH et Fanny RINCK \\ Laboratoire Lidlem, Université Grenoble Alpes, France
}

\section{Résumé}

Inscrite dans le cadre des littéracies universitaires, cette contribution s'attache à la description linguistique d'un phénomène considéré comme un des emblèmes des difficultés que rencontrent les apprentis chercheurs (mastérisants ou doctorants) dans leur initiation à l'écrit de recherche : l'anaphore démonstrative. A partir de la comparaison entre un corpus d'apprenants et un corpus

d'experts, cette étude montre comment ces descriptions linguistiques peuvent être exploitées en situation didactique afin de susciter chez les étudiants des prises de conscience les amenant à mieux repérer et corriger leurs erreurs, et, à terme, à développer leurs compétences rédactionnelles en matière d'écrit scientifique.

\section{Mots clés}

Littéracies universitaires, anaphore démonstrative, compétences rédactionnelles

\section{Introduction}

Notre contribution s'inscrit dans le cadre des littéracies universitaires : nous nous intéressons à l'écrit en lien avec la construction des savoirs et visons une formation linguistique aux genres académiques, fondée sur des descriptions linguistiques en littéracie avancée. La notion de littéracie désigne la diversité des pratiques de lecture et d'écriture, des contextes où elles s'exercent et des compétences qu'elles mobilisent (Barré de Miniac et al., 2004). Empruntée à l'anglais, elle permet de dépasser une vision dichotomique entre l'illettrisme et ce qui n'en est pas. Or, précisément, il s'agit ici de rendre compte d'acquisitions « tardives » dans la maitrise de l'écriture ; la notion de littéracie encourage à adopter une vision selon laquelle il n'y a pas de maitrise aboutie, car on se forme tout au long de la vie à de nouvelles pratiques. Cette notion permet également d'envisager l'écrit comme technologie matérielle et intellectuelle ${ }^{1}$ et de mettre ainsi l'accent sur le fait que l'enjeu pour les étudiants n'est pas seulement de bien écrire.

L'idée que nous défendons est que les descriptions linguistiques servent à élaborer des outils de prise de conscience pour les scripteurs étudiants (et leurs formateurs), basés sur les principes du recours à l'observation de corpus dans le cadre de l'enseignement des langues (Sinclair, 2004); ces outils visent ici spécifiquement à favoriser le repérage de ce qui fonctionne bien ou moins bien dans un texte et à guider ainsi le travail de révision et de réécriture. En d'autres termes, il s'agit de former des scripteurs attentifs à la matérialité de leurs textes, pour qu'ils produisent des écrits mieux maitrisés et pour que l'écriture soit un tremplin pour penser (Rinck et Boch, 2012).

La démarche prend comme point de départ l'identification de difficultés dans les écrits des étudiants et la comparaison avec les pratiques expertes qui servent de référence dans l'écrit académique. Nous nous inscrivons dans une approche intégrative en linguistique de l'écrit,

\footnotetext{
$1 \quad$ "It is key to communication and learning of all kinds and a fundamental condition of access to today's knowledge
} Societies“, UNESCO (2008). The global literacy challenge, http://unesdoc.unesco.org/images/0016/001631/163170e.pdf 
qui va du matériau graphique aux effets pragmatiques. Ainsi, tel signe de ponctuation n'a rien d'anodin et quand on invoque les critères de clarté ou de lisibilité des textes de nos étudiants, il convient d'identifier les assises linguistiques sur lesquelles se fondent nos jugements d'acceptabilité. Si l'on veut aider les étudiants à réécrire et à penser via l'écrit, il faut pouvoir attirer leur attention sur des points d'observation et d'intervention stratégiques.

\section{Objectifs et méthodes}

Les objectifs de notre approche sont d'aider les étudiants et leurs formateurs au repérage de difficultés dans leurs textes. Les difficultés des étudiants dans l'écrit sont étroitement liées à l'appropriation des notions de leur discipline et à la construction d'un raisonnement via l'écrit. Elles se manifestent également par des maladresses d'ordre syntaxique et stylistique ; il ne s'agit pas nécessairement d'erreurs mais de constructions qui peuvent être disqualifiées et/ou être en cause dans l'appréciation du lecteur quant à la cohérence, la clarté ou la qualité du texte. Nous cherchons à identifier précisément à quoi tient un effet général de maladresse avec l'hypothèse didactique qu'un travail à une échelle micro du texte peut aider le scripteur à l'améliorer en profondeur.

Bien entendu, la notion de «maladresse » est subjective, ou du moins culturelle : l'évaluation du degré de maladresse est liée à la communauté de référence du lecteur et à ses propres normes. C'est pourquoi nous comparons les textes d'étudiants aux pratiques dites «expertes » au sens où elles représentent les pratiques de référence dans l'écrit académique. Là encore cependant, on conviendra que la qualité des textes donnera lieu à des appréciations variées.

Nous ne cherchons pas à analyser de manière systématique l'ensemble des difficultés que l'on peut rencontrer dans les écrits des étudiants mais plutôt à identifier des difficultés emblématiques, qui soient des heuristiques pour la description de leurs compétences rédactionnelles et de bonnes entrées pour la formation à l'écrit.

Parmi elles, nous retenons ici la gestion de l'anaphore démonstrative. On sait combien l'interprétation des anaphores en lecture et leur maniement en production sont complexes pour les élèves de primaire ${ }^{2}$, du collège (par exemple Lima et Bianco, 1999), du lycée (Elalouf, 1996) et, pour les étudiants étrangers, (Beguelin, 1988a, b). Dans la visée d'un apprentissage continu de l'écrit, nous ferons donc de l'anaphore une porte d'entrée à nos observations des textes des étudiants, et proposons d'y voir un lieu stratégique pour l'étudiant et son formateur, pour le repérage de maladresses et pour guider la réécriture.

L'anaphore démonstrative a largement été décrite par la linguistique (cf. par exemple Charolles, 1995 ; Corblin, 1998 ; Combettes, 1986 ; Le Pesant, 2002 ; Lundquist et al., 2012, etc.). En tant qu'anaphores, par définition, les anaphores démonstratives sont interprétativement dépendantes d'une portion antérieure du texte. Elles ont une fonction de liaison entre phrases ou propositions et font partie des marqueurs assurant la cohésion du texte, et, du côté du lecteur, sa cohérence. L'enjeu ici est de s'interroger sur ce qui fait qu'une anaphore démonstrative ne trouve pas de référent ou ne peut être interprétée par le lecteur sans un coût cognitif important - autrement dit, sur les caractéristiques linguistiques qui fondent les jugements d'acceptabilité en matière de cohérence textuelle. Nous montrerons aussi en quoi l'anaphore démonstrative peut faire appel à un haut degré de maturité syntaxique et conceptuelle (dans certains cas d'anaphore résomptive en particulier, voir partie 4).

2 L'anaphore est complexe ne serait-ce que dans les aspects proprement morpho-syntaxiques (par exemple, «l'enfant » est repris en « ils », ou inversement). 
D'un point de vue méthodologique, nous avons eu recours à un double corpus :

- d'une part, 50 mémoires de Master 1 (385000 mots) en didactique du français (ressources bibliographiques en linguistique et sciences de l'éducation), produits par des étudiants qui ont le français comme langue première ;

- d'autre part, des écrits de recherche français, publiés ou diffusés en tant qu'écrits de recherche (d'où la désignation en tant qu'écrits « experts »), dans les mêmes domaines que les mémoires du premier corpus :

- 110 articles de linguistique (674000 mots) (Rinck, 2006)

- Des articles, thèses et propositions de communication en linguistique et sciences de l'éducation tirés de la plateforme Scientext : $\underline{\text { http://scientext.msh-alpes.fr }}$

Nous avons mené des analyses quantitatives et qualitatives, sur le nombre d'occurrences des pronoms et déterminants démonstratifs et sur le type d'occurrences dans le corpus des écrits étudiants et dans le corpus «expert »de référence. Nous proposons de montrer comment on peut utiliser ces observations dans la perspective de former les étudiants apprentis-chercheurs (inscrits en master ou en doctorat) au maniement de l'anaphore démonstrative dans les écrits de recherche.

\section{Les anaphores démonstratives pronominales}

3.1. Un usage abondant de « ceci » et « cela » dans les écrits des étudiants

Dans un texte de longueur moyenne ${ }^{3}$, on compte une moyenne de 10 cela dans les écrits des étudiants et moins de $4 \mathrm{chez}$ les experts. Cette différence statistiquement significative se retrouve pour ceci, mais les occurrences sont très faibles (entre 1 et 2 en moyenne par texte). On trouve également des différences dans l'usage de ces derniers/ces dernières, celle-ci, ce qui n'est pas facile à interpréter ${ }^{4}$ mais la prise en compte d'une diversité de marqueurs permet de dire que la sur-représentation de ceci et cela dans les écrits des étudiants n'est pas liée à un usage moindre des autres pronoms - elle peut être liée cependant à un usage moindre de l'anaphore en ce $+\mathrm{N}$, comme on le verra.

\subsubsection{Des effets d'oralité ?}

La forte présence de cela interpelle d'abord d'un point de vue phraséologique; la seule répétition de cet item produit un effet de maladresse. On peut penser qu'il est en partie dû à l'usage dans les écrits des étudiants de traits caractéristiques d'une syntaxe de l'oral.

Cela entre en concurrence avec l'usage de ça, inexistant dans les écrits des experts :

(1) Cela ralentit le rythme de la classe et ça lui demande beaucoup de matériel $(\operatorname{txt} 25)^{5}$

Cela intervient également comme marque d'emphase, en particulier dans des dislocations, et typiquement avec le présentatif «c'est» ou la formule et cela; la question se pose de

\footnotetext{
3 Dans le corpus (écrits étudiants et articles de linguistique) cette longueur moyenne est de 40717 caractères (y compris les espaces), soit un peu plus d'une dizaine de pages.

$4 \quad$ On remarque par exemple dans le corpus considéré une différence statistiquement significative avec celle-ci mais pas avec celui-ci, sans pouvoir l'expliquer.

Nous conservons l'orthographe originale et indiquons en fin d'extrait le numéro du texte dont il est tiré.
} 
l'appréciation du lecteur quant à l'acceptabilité de ce type de constructions dans le cadre d'un écrit académique.

(2) Mais l'écriture est bien plus que cela, c'est également... (txt9)

(3) Découvrir l'écrit, cela passe aussi par... (txt41)

(4) leur compréhension est incomplète ou erronée et cela le lecteur ne s'en rend pas compte (txt 26)

Cette analyse de l'usage de cela en termes d'oralité se manifeste dans des passages spécifiques où les étudiants rapportent les propos d'enseignants qu'ils ont rencontrés lors d'observations qu'ils ont faites en classe, sur leur terrain de stage. On voit ainsi dans leurs écrits leur difficulté à écrire à partir de sources orales ${ }^{6}$ :

(5) Selon elle [l'enseignante], le développement langagier en maternelle c'est fournir aux élèves des outils comme le lexique, la syntaxe ou l'intonation. Seulement elle précise que pour elle ce n'est pas uniquement cela. En effet, selon elle, avoir des outils c'est bien mais il faut surtout avoir des choses à dire. (txt18)

On note ici des marqueurs propres au travail d'écriture à partir de sources (selon elle, elle précise que), et la difficulté à aller au-delà de ce qui serait de la simple transcription (le développement, c'est..., avoir des outils c'est bien mais...) avec des aménagements de surface (cela remplace ça, comme dans ce n'est pas uniquement cela). Un travail de réécriture permettrait de modifier ce qui peut produire un effet de maladresse, sinon spécifiquement un effet d'oralité. L'extrait (5) peut donc être vu comme une étape dans le travail d'écriture, sur le chemin entre un enregistrement ou des notes prises lors de la rencontre avec un acteur de terrain et un écrit académique normé.

\subsubsection{Ressources phraséologiques lacunaires}

Si les étudiants emploient de nombreux ceci et cela, c'est peut-être aussi faute d'exploiter une palette plus large de structures. Ainsi le lecteur parcourant des mémoires peut-il spontanément substituer aux ceci et cela d'autres formulations, et ces marqueurs peuvent alors servir de lieu stratégique pour le scripteur : les repérer doit lui permettre d'envisager des réécritures.

C'est le cas notamment lorsque ceci et cela s'inscrivent dans des contextes cumulant diverses difficultés d'ordre interpropositionnel. Ainsi, en (6), avec l'usage successif de deux participes présents (les enfants ayant..., cela permettant):

(6) L'auteur explique que même si les livres ont toujours été présents à l'école, ils prennent à présent une place particulière, les enfants ayant eux-mêmes envie d'écrire leurs premières histoires, cela permettant «les premières expériences gratifiantes » $(\mathrm{txt} 4)$

On voit ici que l'accompagnement à l'écrit doit mobiliser de multiples entrées linguistiques ; outre les anaphores, un travail sur les participes présents pourrait représenter une voie intéressante, pour trouver d'autres formulations face aux contraintes de linéarité et de hiérarchie des constituants de la phrase. Les deux-points par exemple peuvent servir à

$6 \quad$ Cela a été montré dans d'autres genres que les mémoires d'étudiants, par exemple dans la sphère des écrits professionnels, quand les étudiants ont par exemple à écrire un compte rendu de réunion (Rinck et Sitri, 2012). 
marquer une relation d'étayage après une première proposition, tout en permettant de faire l'économie d'une explicitation plus poussée des liens logiques entre propositions (justification, illustration?).

Dans certains cas, la recherche d'autres formulations peut consister en des substitutions terme à terme, au profit de variantes moins maladroites ou plus prestigieuses aux yeux d'un lecteurévaluateur en contexte académique.

Ainsi on pourra suggérer la formule c'est ainsi que dans :

(7) C'est comme cela que l'enfant parviendra « à enrichir son vocabulaire (txt18)

Ou une formule comme il s'agit de dans :

(8) Nombre d'observateurs pensaient qu'il était nécessaire d'enseigner une grande quantité de mots (...). Or, comme le souligne A. Florin (1999), cela est une erreur dans le sens où,... (txt20)

ou encore dans :

(9) L'orthographe connote, dans certains esprits, la valeur de beauté. Cela était la perception de l'orthographe de Flaubert (txt53)

l'usage de tel (telle, en l'occurrence), qui fait d'ailleurs partie des marqueurs que S. Garnier et A. Savage (2011) proposent de travailler auprès de publics qui apprennent le français écrit à un niveau avancé, au titre de marqueurs sous-employés par ces publics.

Dans le même sens, on pourra faire porter l'attention sur pour cela dont on trouve 122 occurrences dans le corpus des écrits des étudiants contre 18 chez les experts ${ }^{7}$. Cet usage surabondant est en lui-même le signe d'une maladresse et la recherche d'autres formulations impose une plus grande explicitation (par exemple, dans une formule comme « c'est pour cela que », vise-t-on l'expression d'une cause ? d'un but?).

\subsection{De la phrase au texte : les mentionnels}

Comme ceci et cela, les mentionnels celui-ci et ce dernier appellent des remarques sur la cohésion textuelle et notamment le lien entre phrases ou propositions, et sur ce qui peut poser problème au lecteur en termes de cohérence ou de qualité du texte de manière générale.

Leur propriété est de permettre une désambiguïsation en cas de double mention, autrement dit en présence de deux candidats à la référence, car ils sélectionnent le candidat sur un critère de proximité ou de contiguïté (Corblin, 1998).

D'abord, la forte présence de ce type de pronoms peut donner lieu à des considérations d'ordre stylistique, sur l'effet de lourdeur qu'ils produisent en eux-mêmes ou par leur accumulation. Une telle remarque fait écho à une autre considération stylistique, selon laquelle il faut éviter les répétitions lexicales; on peut penser en effet que les étudiants ont bien (ou trop bien) intégré cet interdit attaché aux répétitions alors que la répétition terme à terme est sans doute parfois plus productive que l'usage d'un substitut pronominal.

\footnotetext{
$7 \quad$ Nous donnons ici les fréquences absolues mais le corpus des écrits des étudiants est deux fois moins
} grand, ce qui augmente encore la différence avec les écrits des experts. 
C'est le cas dans l'extrait (10), où sur une petite fiche est repris par sur celle-ci plusieurs lignes plus loin, dans le paragraphe suivant ${ }^{8}$ :

(10) Le travail individualisé, appelé TP, est présenté sur une petite fiche distribuée tous les quinze jours à l'élève. Les TP sont des séances de travail personnalisé dans lesquelles les élèves on [sic] un programme personnalisé.

La professeur des écoles annonce sur celle-ci la leçon à réviser (txt47)

Par ailleurs, les mentionnels celui-ci et ce dernier sont souvent utilisés sans que cela se justifie ; ils interviennent sans qu'on ait affaire à deux candidats à la référence, comme dans les exemples suivants :

(11) Je vais donc m'appuyer sur la méthode inductive et les bienfaits de celle-ci pour élaborer une méthode (txt53)

(12) Nous sommes donc parties du principe qu'une activité préalable autour du lexique serait nécessaire à toute autre activité utilisant ce dernier (txt18)

(13) C'est-à-dire que l'enfant va tenter de deviner le mot dans sa globalité et non pas décoder ce dernier en l'analysant. (txt10)

La consigne pourrait alors être de trouver des reprises plus «simples »: le déterminant possessif dans l'ex. 11 (la méthode et ses bienfaits) ou le pronom personnel dans les ex. 12 (le lexique et une activité l'utilisant) et 13 (deviner le mot et le décoder).

Dans l'usage de l'anaphore démonstrative pronominale, ce qui heurte le lecteur et peut être jugé comme un effet de lourdeur représente un coût cognitif important. L'anaphore déclenche la recherche du référent et le coût est d'autant plus élevé que l'on a un éloignement du référent et des ambiguïtés référentielles. C'est notamment le cas avec cela que l'on peut qualifier à l'instar de M.J. Béguelin (1988b : 78) de pronom «à large spectre ». L'exemple suivant fournit une bonne illustration de cette propriété linguistique et par voie de conséquence du coût cognitif imposé au lecteur :

(14) Effectivement, lors de la lecture du texte nous pouvons supposer que de nombreux parents mettent une intonation particulière au texte et que celle-ci permet à l'enfant de se rappeler des passages. Cela se ressent car lorsqu'ils lisent ils mettent un ton particulier à certaines phrases. Les livres choisis sont d'ailleurs des supports propices à cela,... (txt22)

Le pronom démonstratif est alors le signe d'un manque d'explicitation appelant un travail de réécriture. C'est ce que l'on remarque également à travers l'usage d'anaphores démonstratives prenant la forme de reprises nominales en ce $+\mathrm{N}$.

\section{L'anaphore démonstrative en ce $+\mathrm{N}$}

\subsection{Préalables}

Le cas de l'anaphore démonstrative (c'est-à-dire introduite par un déterminant démonstratif de type ce, cette, cet, ces) est bien décrit en linguistique. On distingue classiquement (Kleiber, 1986) :

\footnotetext{
$8 \quad$ Notons qu'ici le lecteur est guidé par la mise en gras !
} 
- L'anaphore fidèle, qui désigne un syntagme nominal (N2) dont la tête est identique à celle de l'antécédent (N1) :

(15) Il faut pouvoir comparer les objectifs de chacune des deux activités au départ et les résultats finaux par rapport à ces mêmes objectifs. (txt 18)

- L'anaphore infidèle, entendue ici comme reprise par synonyme ou quasi synonyme, hyperonyme ou métonymie ${ }^{9}$. La tête est différente de celle de l'antécédent, mais N1 et N2 évoquent tous deux le «même objet de pensée » (Blanche-Benveniste \& Chervel, 1966 : 31).

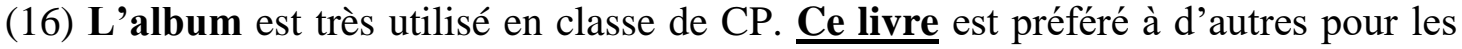
raisons suivantes $(\ldots)($ txt 12$)$

- L'anaphore résomptive qui résume une portion du discours : N2 ne renvoie plus à un N1 clairement identifié, mais à un empan textuel variable, qui peut aller de quelques lignes (comme en (17)) au paragraphe.

(17) D'après Totereau (2010), vers 3-4 ans, les enfants acquièrent un vocabulaire de plus en plus abondant (1000 mots), produisent des phrases de plus en plus longues et complexes, manient correctement la première personne du singulier et commencent à employer un vocabulaire traduisant les émotions et les sentiments.

Cette extension du vocabulaire est possible, selon Totereau (2010, cours de psychologie du développement), grâce à (...) (txt 19)

Ce sont les cas d'anaphore infidèle et résomptive qui vont nous occuper ici : ils sont souvent peu maitrisés par les étudiants alors qu'ils représentent un exemple intéressant d'outils linguistiques au service de la construction du texte envisagé comme un tout cohérent.

La réflexion métalinguistique autour de ces outils peut être utile pour améliorer les textes et favorise de notre point de vue une prise de conscience fondamentale concernant l'écriture chez les étudiants : écrire aide à penser, et penser permet de mieux écrire. L'anaphore démonstrative illustre en effet l'articulation entre la dimension linguistique et la dimension cognitive que met en jeu l'écriture : si la présence d'un «cela » doit interroger, c'est parce que la question de son référent surgit et avec elle celle de la construction du sens au fil du texte. Les anaphores jouent sur la cohérence du texte aux yeux du lecteur et nécessitent de la part du scripteur une conscience fine du déroulement de son raisonnement. Les anaphores infidèles et résomptives sont tout particulièrement concernées et elles impliquent des compétences lexicales et une bonne connaissance du domaine de spécialitét ${ }^{10}$.

Comme nous allons le montrer, cette réflexion métalinguistique peut être provoquée chez les étudiants par l'observation d'exemples d'anaphores infidèles et résomptives produites par leurs pairs et considérées comme inappropriées ou partiellement inappropriées.

\footnotetext{
9 Nous mettons volontairement de côté les autres cas inventoriés par certains linguistes comme ReichlerBeguelin (1995), qui inclut dans la classe des anaphores infidèles l'anaphore résomptive, qu'on traitera ici à part, en ce qu'elle ne relève pas de la même opération métalinguistique : dans un cas il s'agit de substituer un terme à un autre, dans l'autre de résumer un empan textuel - par exemple un paragraphe - en une expression.

$10 \quad$ Nous ne discuterons pas ici de la distinction ni des relations entre anaphores résomptives et conceptuelles, et nous en tiendrons aux premières ; la terminologie varie en effet beaucoup d'un auteur à un autre, les anaphores conceptuelles pouvant être considérées comme un cas d'anaphores résomptives ou l'inverse.
} 


\subsection{Une démarche didactique fondée sur l'observation de corpus de pairs}

Ainsi, suite à une définition rapide des trois cas d'anaphore (telle que présentée ci-dessus), le formateur peut inviter ses étudiants à identifier dans un corpus d'exemples ${ }^{11}$ les reprises anaphoriques en ce $+\mathrm{N}$ et à analyser leur degré de pertinence.

(18) A partir de nos observations de classe, nous avons notamment retenus deux dispositifs : la méthode de lecture «La planète des Alphas » et les ateliers d'aide à la lecture appelés Club Coup de Pouce CLE. Nous présenterons dans un premier temps l'état des lieux théoriques de ces deux procédés. Nous n'omettrons pas d'inclure dans cette partie les instructions officielles (IO) concernant l'apprentissage de la lecture en Grande Section et au Cours Préparatoire afin de pouvoir situer ces dispositifs par rapport aux compétences demandées et attendues. (txt 14)

(19) Le langage dit en "situation", appelé aussi langage "d'action" ou encore de "communication immédiate" est surtout utilisé par l'enseignant en classe de petite section de Maternelle. Ce langage est un repère pour le tout jeune enfant car il ne maîtrise pas suffisamment le vocabulaire et sa langue maternelle en général. Ce parlé [sic] fait référence à un langage factuel car il est ancré dans une situation partagée par les interlocuteurs qui échangent dans le moment présent de l'action. (txt 20)

(20) (A propos de la grille EVA) Selon P. Sève, ce tableau a apporté un regard normatif sur la production d'écrit. Pour que ce modèle d'évaluation soit efficace, des critères doivent être sélectionnés en relation avec le projet d'écriture, les connaissances des enfants... et permettre ainsi de clarifier les points d'intervention de l'enseignant. Cette sélection de critères facilite également l'utilisation de cette grille qui peut être fastidieuse. Cependant ce modèle «s'intéresse trop au texte » (...). (txt 11)

(21) C'est sur la base du langage en situation que l'on acquiert un langage d'évocation, il permet d'anticiper des faits ou bien de revenir sur certains événements déjà passés. Ce processus du langage commence à s'installer dès l'âge de 3 ans. (txt 20)

(22) Le travail scolaire se fait au moyen de brochures ou de fiches détaillées dans lesquelles les élèves trouvent toutes les indications sur le travail qu'ils ont à accomplir. Un contrôle est fait à tous les niveaux du travail. Ce système a suscité l'enthousiasme (...). (txt 21)

(23) Lire suppose donc de réaliser des traitements de différents niveaux : «signification des mots, interprétation des groupes de mots, intégration des informations nouvelles aux informations anciennes » (Golder \& Gaonac'h, 1998: 86). Cette représentation suppose donc diverses connaissances et mobilise plusieurs processus cognitifs que le lecteur devra coordonner. (txt 27)

\footnotetext{
11 Les exemples du corpus proposé ici étant extraits de mémoires de Master d'étudiants se destinant au métier d'enseignant, ils sont particulièrement adaptés à un public ayant un profil voisin, et ne sont fournis qu'à titre d'illustration de la démarche. Dans le cas de publics très éloignés de ce profil, l'élaboration d'un corpus ad hoc devient bien évidemment nécessaire. Dans tous les cas, il est à nos yeux préférable de constituer un corpus à partir des productions émanant du groupe d'étudiants à qui est proposée la formation à l'écriture universitaire.
} 
L'observation de ce corpus peut être orientée de manière à déclencher des débats d'ordre métalinguistique portant sur les thématiques développées ci-dessous.

a) Les limites de la typologie :

(18), (19) et (20) relèvent plutôt de l'anaphore infidèle (avec des cas d'alternance anaphore fidèle/anaphore infidèle) tandis que (21), (22) et (23) illustrent plutôt des cas d'anaphore résomptive ; cela dit, certains exemples sont délicats à identifier et montrent la porosité entre les catégories d'anaphore (et au-delà, les limites de toute typologie). Si l'on peut considérer qu'en (19) N2 ( «ce langage ») peut être considéré comme une reprise hyperonymique de N1 («Le langage dit en "situation", appelé aussi langage "d'action" ou encore de "communication immédiate" »), interprétation induite par la présence du déterminant démonstratif « ce », que faire des reprises nominalisées en N2, comme en (20) ? L'anaphore "cette sélection de critères » est-elle infidèle, si l'on considère qu'elle reprend simplement « des critères doivent être sélectionnés », ou résomptive, si la reprise s'étend à ce qui suit («en relation avec le projet de l'écriture, les connaissances des enfants... ») ? Les réponses à ces questions sont bien entendu moins importantes que la discussion qu'elles provoquent, l'objectif étant de mettre à distance les phénomènes linguistiques pour en faire des objets d'analyse et par là aiguiser le regard métalinguistique des étudiants.

\section{b) Eviter la répétition à tout prix ?}

Dans les écrits d'étudiants, on a le sentiment que les anaphores infidèles sont parfois liées à la volonté de ne pas répéter N1. (25), (26) et (27) illustrent cette contrainte stylistique, sans doute héritée du lycée, que semblent s'imposer les étudiants (comme déjà évoqué avec l'utilisation abusive des mentionnels tels que ce dernier ou celui-ci). Or, on peut attirer leur attention sur la pertinence d'une telle contrainte dans ces exemples (et plus généralement dans l'écrit de recherche, où la redondance caractérise le travail de conceptualisation, notamment dans le respect de la terminologie), où la recherche d'une anaphore s'apparente à une recherche gratuite de synonymes et génère des maladresses qu'une simple répétition aurait pu éviter ; (27) est de ce point de vue caricatural : si on peut considérer que tableau est repris de manière pertinente par modèle d'évaluation, en ce que cette anaphore intègre l'idée du regard normatif qu'il [le tableau] apporte à l'évaluation, le terme tableau est ensuite repris par grille, puis encore par modèle (anaphore fidèle tronquée ?) en l'espace de quelques lignes, et cette chaine anaphorique représente plus un frein qu'une aide à la compréhension du lecteur. Plus ennuyeux (car nous basculons de la maladresse à la non pertinence), en (26), ce parlé («ce parler »?) ne peut constituer une reprise anaphorique pertinente de langage en situation (mais peut-être l'auteur ignore-t-il le sens de parler en linguistique et a-t-il trouvé le terme pertinent au regard du caractère oral des échanges spontanés qu'implique la notion de langage en situation?). Enfin, en (25), procédés apparait entre deux occurrences identiques (dispositifs), mais n'est pas à même, à nos yeux, de requalifier le référent de dispositif dans ce contexte. L'hypothèse d'une simple recherche de synonyme plutôt que d'une anaphore infidèle est ici renforcée par le fait qu'on trouve "procédé » comme synonyme de « dispositif » dans le dictionnaire de synonymes en ligne Crisco, dont on peut supposer qu'il fait partie des ressources conseillées aux étudiants et qu'ils utilisent. Cela dit, le terme même de dispositif semble peu approprié dans la mesure où il renvoie d'une part à méthode de lecture (qui peut être considérée comme un outil pédagogique, un manuel) et d'autre part à atelier d'aide à la lecture (qui est un véritable dispositif didactique). 
La discussion peut ainsi porter sur la difficulté de recruter une anaphore hyperonyme lorsque les deux N1 ne se situent pas tout à fait sur le même plan sémantique, et, au-delà, sur le rôle même de l'anaphore, qui ne doit pas être considérée comme une reprise synonymique visant à éviter la répétition, mais comme une plus-value au plan de la cohésion textuelle et de la progression thématique.

c) De l'intérêt de l'anaphore résomptive en tête de phrase

Les exemples (21) à (23) illustrent des cas où l'anaphore résomptive se situe en tête de phrase ; on peut interroger les étudiants sur l'intérêt d'une telle structure, et les amener à considérer que l'anaphore revêt ici une double fonction : elle assure à la fois la continuité du raisonnement (via la reprise de l'objet de discours), mais aussi sa progression (via la sélection d'un attribut de cet objet de discours), qui du fait de sa place en tête de phrase entraine le développement d'une prédication nouvelle par topicalisation.

d) Difficulté à manier le lexique transdisciplinaire et le lexique de spécialité

Les étudiants éprouvent parfois de la difficulté à utiliser le lexique adéquat pour préciser par écrit leur pensée, et les anaphores résomptives manifestent souvent cette difficulté ; (21) en est un bon exemple, avec l'anaphore résomptive ce processus de langage, peu appropriée ici (et à laquelle on substituera la simple répétition de N1 : «Le langage d'évocation »).

En (22) et (23), on n'observe pas à proprement parler de problème dans le choix des termes ; on peut noter toutefois que les termes représentation et système dans les anaphores résomptives sont utilisés seuls; or, on peut amener l'étudiant à développer sa conscience du fonctionnement de l'écrit de recherche en le sensibilisant à la pertinence d'accompagner le nom de l'anaphore d'un modifieur (adjectif, complément du nom ou proposition relative) qui peut fournir au lecteur des informations supplémentaires sur la posture qu'il adopte, autrement dit sur le point de vue qu'il est en train de construire.

\subsection{L'anaphore résomptive chez les experts}

Le travail qui précède peut être complété par un aperçu d'anaphores résomptives de type $\mathrm{Ce}+$ Nom + prép + Nom, a priori très exploité par les experts ${ }^{12}$; le tableau suivant en fournit quelques exemples :

\begin{tabular}{|c|c|}
\hline Patron syntaxique $\mathrm{Ce}+\mathrm{N}+$ Pre & Verbe \\
\hline Ce type de mise en activité est & Ce cas de figure est \\
\hline Ce point de vue implique & Cet ensemble de propositions est \\
\hline Ces modèles de référence sont & $\begin{array}{l}\text { Cette diversité de manifestations } \\
\text { confirme }\end{array}$ \\
\hline
\end{tabular}

12 Cf. les travaux de Tutin (2007) et les statistiques présentées sur la page http://scientext.mshalpes.fr/scientext-site/spip.php?article39. 
Cette vue d'ensemble souligne

Ce problème de représentativité est
Ce changement de perspective

permet

Cet aperçu des résultats invite

Ces éléments de preuve sont
Cette prise de conscience représente

Cet ensemble de contraintes limite
Cette vision des choses a

Tableau 1 : Anaphores résomptives en $\mathrm{Ce}+\mathrm{N}+$ Prép + Nom

L'observation de ces exemples peut déclencher ici encore une discussion productive sur la faible représentativité de ce patron linguistique dans leurs écrits alors qu'il est très exploité chez les experts ${ }^{13}$. Au-delà du caractère routinier de ce lexique transdisciplinaire ${ }^{14}$ dans l'écrit scientifique, nécessairement encore méconnu des apprentis-chercheurs, on peut supposer que les étudiants n'ont pas encore acquis la maturité intellectuelle qui nous semble requise par la sélection de ce type d'anaphore : identifier qu'il est question de «point de vue », de «prise de conscience », de « vision des choses » ou encore de «changement de perspective » implique un décentrage et un recul important par rapport à la réflexion théorique dont ils doivent rendre compte alors qu'ils découvrent à peine le foisonnement du champ. Circonscrire un " point de vue » par exemple suppose en effet d'avoir conscience qu'il en existe plusieurs, et de distinguer, au niveau épistémique, ce qui a statut de point de vue, d'acquis dans un domaine, etc. Cela étant, il nous semble primordial d'entrainer les étudiants à reconnaitre et à pratiquer les anaphores résomptives, de manière à les amener peu à peu à mieux organiser et s'approprier l'état de la réflexion théorique qu'ils donnent à voir.

Dans cette phase d'observation du fonctionnement de l'anaphore résomptive, on peut également amener les étudiants à mieux saisir comment cet outil linguistique peut de manière économique et efficace servir l'argumentation, a fortiori si le groupe nominal est associé à un modifieur (un adjectif, un complément du nom, ou encore une proposition relative). Ainsi, en (24), l'emploi du démonstratif « ce » (ce codage) signale au lecteur que le groupe nominal est un point nodal de son discours (Lundquist et al., 2012), tandis que le nom et l'adjectif évaluatif qui lui est associé ( «ce codage efficace») fournissent des informations sur la manière dont l'auteur résume et interprète la portion de texte reprise dans l'anaphore résomptive :

Le formalisme IDAREX (Breidt \& Segond 1995) permet ainsi de coder les variantes sous la forme d'expressions régulières. Par exemple, (...). Ce codage efficace permet de rendre compte de toutes les variations observées, mais apparaît malgré tout assez lourd (...) [Corpus scientext, TAL]

Les anaphores résomptives avec nom et modifieur, dont en particulier les adjectifs évaluatifs, sont très fréquents chez les experts, contrairement aux représentations que se font

\footnotetext{
13 A titre d'exemple, « ce point de vue » est utilisé 37 fois dans le corpus d'articles et seulement 3 fois dans le corpus de mémoires de master.

14 Par «lexique transdisciplinaire» (ou «lexique épistémique »), on entend le lexique conceptuel caractéristique de l'écrit de recherche (principe, méthode, hypothèse, problématique, résultats, etc.) et transversal aux disciplines (cf. par exemple Tutin, 2007).
} 
généralement les étudiants de l'écrit de recherche comme étant exempt de toute trace de subjectivité; on en trouvera dans le tableau 2 quelques illustrations (issues du corpus Scientext) :

\begin{tabular}{ll} 
Ce codage efficace permet & Cette restriction brute laisse \\
\hline $\begin{array}{l}\text { Cet ouvrage complexe mêle } \\
\text { Cette question importante est }\end{array}$ & Ce point particulier est \\
Ce fonctionnement dynamique est & Ce comportement maladroit est \\
Ces méthodes subjectives sont & Ces définitions uniformes soulignent \\
Ces principes fondamentaux rendent Ce graphique complexe visualise \\
Ce problème majeur est & Ces points négatifs relèvent
\end{tabular}

Tableau 2: Un patron syntaxique productif chez les experts : $\mathrm{Ce}+\mathrm{N}+$ Adjectif + Verbe

En outre, l'observation de ce type d'énoncés peut permettre aux étudiants de mieux assimiler l'une des contraintes fortes - et souvent source de malentendus ${ }^{15}$ - de l'écrit de recherche, qui consiste à construire un point de vue. Intégré à l'anaphore résomptive, le modifieur peut avoir une valeur argumentative en guidant le lecteur dans la perspective adoptée par l'auteur de l'énoncé, guidage souvent mal assuré par les étudiants peu conscients de cette caractéristique de l'écriture universitaire (et de l'écriture de recherche en particulier).

\section{Bilan}

Tout au long de cette étude portant sur l'anaphore démonstrative, nous avons cherché à proposer des activités recourant à l'observation de corpus (de pairs ou d'experts) susceptibles selon nous de favoriser certaines prises de conscience nécessaires à la construction d'une représentation fine du fonctionnement de l'écriture, et en particulier de l'écriture de recherche. L'encadré ci-dessous pointe quelques-unes de ces prises de conscience, qui, idéalement, devraient pouvoir être produites par le groupe d'étudiants concerné par ces activités :

Reformuler un cela peut être rentable : face à un cela, s'interroger sur sa pertinence ;

$>$ La répétition n'est pas à bannir à tout prix : répéter ou reformuler ? Ou comment concilier idéal de clarté et qualité stylistique ;

$>$ Le choix réfléchi de l'anaphore (même si l'opération est coûteuse cognitivement) est une aide à l'élaboration du raisonnement et favorise sa réception auprès du lecteur ;

$>$ Spécifier l'anaphore (avec un adjectif par exemple) permet d'orienter économiquement le discours et de marquer un point de vue sur l'objet dont on parle.

Les constats que nous avons formulés ici sur les difficultés des étudiants dans l'usage des anaphores démonstratives et dans la prise en charge de la cohérence textuelle en français langue maternelle sont en phase avec les observations faites sur les acquisitions en langue seconde (voir par exemple Bartning et Kirchmeyer, 2003 ; Bordet, 2011). On sait par ailleurs

$15 \quad$ Cf. Rinck \& Boch (2012). 
que les anaphores représentent une difficulté cruciale pour les publics dits peu littéraciés et on voit ici qu'il s'agit d'une compétence clé et nécessairement complexe pour le scripteur, y compris chez des adultes diplômés :

«Préconiser une introduction explicite des objets-de-discours et un recours minimal aux implicitations, cela revient à réduire la fréquence des situations de calcul inférentiel au cours du processus interprétatif. Ce faisant, il faut savoir que l'on

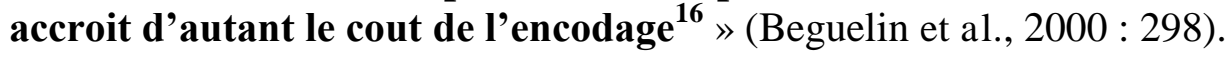

Le champ des littéracies universitaires a pour objet les problèmes spécifiques que pose aux étudiants une écriture mobilisant les savoirs disciplinaires et qui se veut une écriture pour penser. Il questionne en cela aussi les compétences avancées à l'écrit et montre l'intérêt de mener des études sur les acquisitions langagières au-delà des âges tardifs de l'enfance et pas seulement en langue seconde.

La linguistique de l'écrit et les analyses des textes et des discours permettent de donner des assises solides aux discours et dispositifs d'accompagnement à l'écrit. Elles peuvent éclairer les difficultés des étudiants, permettre aux formateurs de s'interroger eux-mêmes sur leurs jugements d'acceptabilité et favoriser un meilleur guidage dans la réécriture.

L'analyse de textes "déviants » n'est pas qu'un domaine d'application de la description linguistique. Si celle-ci revendique le fait d'être basée sur les usages, elle tend parfois à perpétuer l'idée de compétences propres à des situations de communication «idéales », qui se caractériseraient par la cohérence des textes et leur clarté. Les écrits des étudiants mettent en doute ce que H. Nølke présente comme un axiome, celui selon lequel « Tout texte est cohérent. Par définition. C'est là un axiome qui dirige toute interprétation de texte » (Nølke, 1994 : 259). Sauf à les qualifier de «non-textes » tant ils sont parfois maladroits ou confus, ils interpellent leur lecteur et invitent à une réflexion sur le processus par lequel le texte, plutôt que d'être a priori conçu comme un tout cohérent, peut se construire comme tel.

\section{Bibliographie}

APOTHELOZ, D., REICHLER-BEGUELIN, M.-J., 1995, «Construction de la référence et stratégies de désignation », Tranel, 23, p. 227-271.

BARRE-DE MINIAC, C., BRISSAUD, C., RISPAIL, M. (dir.), 2004, La littéracie. Conceptions théoriques et pratiques d'enseignement de la lecture-écriture, Paris, L'Harmattan.

BARTNING, I., KIRCHMEYER, N., 2003, «Le développement de la compétence textuelle à travers les stades acquisitionnels en français L2 », Acquisition et interaction en langue étrangère, Aile, 19, < http://aile.revues.org/1112>.

BEGUELIN, M.-J., MATTHEY, M., BRONCKART, J.-P., CANELAS-TREVISI, S., 2000, «Anaphores pronominales et lexicales », dans M.-J. Beguelin (dir.), De la phrase aux énoncés : grammaire scolaire et descriptions linguistiques, Paris, Duculot, p. 289-306.

BLANCHE-BENVENISTE, C., CHERVEL, A., 1966, «Recherches sur le syntagme substantif $»$, Cahiers de lexicologie, 9, p. 3-37.

[REICHLER-]BÉGUELIN, M.-J., 1988a, «Anaphore, cataphore et mémoire discursive », Pratiques, 57, p. 15-43.

16

C'est nous qui soulignons. 
[REICHLER-]BÉGUELIN, M.-J., 1988b, Écrire en français. Cohésion textuelle et apprentissage de l'expression écrite, Neuchâtel-Paris, Delachaux et Niestlé, 1988, 2e édition, 1990.

BORDET, G., 2011, «'This' comme marqueur privilégié du genre : le cas des résumés de thèses », Discours $9,<$ http://discours.revues.org/8506>.

CHAROLlES, M., 1995, «Comment repêcher les derniers? Analyse des expressions anaphoriques en ce dernier », Pratiques, 85, p. 89-112.

COMBETTES, B., 1986, «Introduction et reprise des éléments d'un texte », Pratiques, 49, 69-84.

CORBLIN, F., 1998, « Celui-ci anaphorique : un mentionnel », Langue Française, 120, p. 3343.

ELALOUF, M.-L., 1996, « La maîtrise de la reprise anaphorique au lycée », Linx, 8, p. 71-90

GARNIER, S., SAVAGE, A., 2011, Rédiger un texte académique en français, Paris, Orphrys.

KLEIBER, G., 1986, «Adjectif démonstratif et article défini en anaphore fidèle », dans J. David, G. Kleiber (dir.), Déterminants, syntaxe et sémantique, Paris, Klincksieck, p. 169-185.

LE PESANT, D., 2002, «La détermination dans les anaphores fidèles et infidèles », Langages, 145 , p. 39-59.

LIMA, L., BIANCO, M., 1999, «Comprendre et interpréter les références pronominales dans des histoires : le cas de 'il' et de 'lui' », Revue française de Pédagogie, 126, p. 83-96.

LUNDQUIST, L., MINEL, J.-L., COUTO, J., 2012, «La navigation discursive. L'anaphore résomptive et mouvement discursif », dans F. Pugnière-Saavedra, F. Sitri, M. Veniard (dir.) L'analyse du discours dans la société, Paris, Honoré Champion, p. 365-389.

NØLKE, H., 1994, Linguistique modulaire : de la forme au sens, Louvain-Paris, Peeters.

RINCK, F., 2006, Figure de l'auteur et identité disciplinaire du genre. L'article de recherche en sciences du langage et en lettres, Thèse de doctorat, Université Stendhal Grenoble 3.

RINCK, F., BOCH, F., 2012, «Enunciative Strategies and Expertise Levels in Academic Writing: How do Writers Manage Point of View and Sources ? », dans M. Castelló, C. Donahue (dir.), University Writing: Selves and Texts in Academic Societies, Bingley-UK, Emerald Group Publishing, p. 111-128.

RINCK, F., SITRI, F., 2012, «Pour une formation linguistique aux écrits professionnels », Pratiques, 153-154, p. 71-83.

TUTIN, A., 2007, (éd.). «Lexique et écrits scientifiques », Revue Française de Linguistique Appliquée, 2, Amsterdam, De Werelt. 